\title{
FACTORS INFLUENCING THE SUCCESSFUL OUTCOME AND RESPONSE IN STRABISMUS SURGERY
}

\author{
OZLEM EVREN ABBASOGLU, EMIN CUMHUR SENER and ALI SEFIK SANAC \\ Ankara, Turkey
}

\begin{abstract}
SUMMARY
In this retrospective study based on 140 esotropic and 51 exotropic patients, the factors influencing successful outcome and response to strabismus surgery were investigated. Thirteen independent variables were chosen. The pre-operative deviation was found to be the only discriminant factor for early and late successful surgical outcomes in esotropic patients. For exotropic patients the visual acuity of the left eye was the discriminant factor for early successful surgical outcome. In esotropic patients the response to surgery increased with increasing amounts of pre-operative deviation. It was lower for patients with older age of onset and larger amounts of medial rectus recession. For exotropic patients the response to surgery was higher for larger pre-operative deviations. Eliminating possible sources of error when determining the preoperative deviation will improve the predictability of the response to surgery and surgical outcome.
\end{abstract}

The aim of strabismus surgery is to straighten the eyes in the primary position and to improve versions and the range of binocular single vision. The outcome of strabismus surgery shows great variability among patients. The determination of preoperative and intraoperative factors affecting successful surgical outcome would help us to be more confident when planning a surgical intervention. The predictability of the surgical outcome also depends on the assessment of factors affecting the response to strabismus surgery. von Graefe ${ }^{1}$ was the first to mention the dose-response relationship in strabismus surgery in 1857 and von Pflugk ${ }^{2}$ followed him using the degree/mm ratio (squint-angle reduction per millimetre of surgery). Many surgical formulas have been proposed, on the basis of both clinical experience and artificial eye models, to detect factors affecting the response to surgery and to improve the predictability of strabismus surgery. ${ }^{3-8}$ Controversy

Correspondence to: Ali Sefik Sanac, MD, Hacettepe University School of Medicine, Department of Ophthalmology, Sihhiye Ankara, 06100 Turkey. still exists regarding the amount of surgery appropriate for different angles of strabismus.

This study was undertaken to determine the preoperative variables that can enable us to predict a successful outcome and to plan the most appropriate surgical intervention.

\section{PATIENTS AND METHODS}

We reviewed the case notes of consecutive patients undergoing horizontal recess-resect surgery for comitant esotropia and exotropia at Hacettepe University between 1984 and 1994. Our esotropic patients consisted of those with acquired esotropia (deviation recognised after 6 months of age as indicated by parental history or confirmed by an ophthalmologist) and exotropic patients included patients with intermittent and constant exotropia. Our specific exclusion criteria eliminated patients whose surgical procedure involved symmetrical medial rectus recession surgery for esotropia and lateral rectus recession surgery for exotropia, adjustable suture technique and simultaneous oblique muscle surgery. We included patients on whom we performed simultaneous vertical transposition of horizontal recti for treatment of $\mathrm{A}$ or $\mathrm{V}$ pattern. We have previously shown that simultaneous vertical transposition of horizontal recti does not have any effect on the outcome of horizontal recess-resect surgery for the same patient group. ${ }^{9}$ Also Kutschke and $\mathrm{Keech}^{1 \boldsymbol{\theta}}$ showed that the vertical displacement of horizontal muscles did not significantly affect the amount of horizontal correction achieved. In addition, we excluded patients with ocular pathology such as nystagmus, microcornea, a history of previous strabismus surgery, botulinum toxin injection, paralytic strabismus and mechanical restrictions as determined by forced duction testing.

Patient data were analysed to determine the factors influencing surgical success and response to surgery. Patients were assigned to one of the following surgical outcome groups on the basis of 
their post-operative deviations: (1) success; (2) residual esotropia/exotropia. A successful outcome was defined as final alignment within \pm 5 degrees of straight, with or without evidence of binocular single vision. ${ }^{11-15}$ Residual esotropia was defined as a deviation of more than 5 degrees of esotropia, residual exotropia as a deviation of more than 5 degrees of exotropia. Patients with a post-operative deviation below 10 degrees of orthotropia were considered cosmetically successful. Factors influencing early and late surgical success were analysed by the discriminant analysis method. For assessment of early surgical success, measurements taken in the sixth post-operative week were used for analysis. Late surgical outcomes were analysed for patients who had at least 1 year of follow-up. As patients come from a distance, the last post-operative measurements were taken for data analysis.

Discriminant analysis is a statistical method used to determine the independent variables that are predictors of an outcome group. Surgical success was defined as the outcome group in this study. A computer-assisted program (SPSS) was used for discriminant analysis. In this program a stepwise analysis of variance was performed.

In the second part of the study we investigated the factors influencing the response to surgery. The response to surgery was defined as the change in deviation divided by the amount of surgery. The change in deviation is the absolute change in deviation from pre-operative to post-operative measurement. The amount of surgery is the sum (in millimetres) of the recession and resection performed. The data were analysed with a stepwise multiple regression analysis.

For the two studies the same independent variables for each case were selected: age of onset of deviation (years), age at surgery (years), interval between onset and surgery (years), pre-operative deviation (degrees), refractive error of the right and the left eye (spherical equivalent as dioptres), anisometropia (the difference between the spherical equivalent values of refractive errors of the right and the left eye in dioptres), visual acuities of the right and the left eye, presence of amblyopia (patients were divided into three groups according to presence and depth of amblyopia, as discussed later), medial rectus surgery (recession or resection, in millimetres), lateral rectus surgery (recession or resection, in millimetres), presence of alphabetical pattern.

We measured the angle of strabismus with both the alternate prism cover test and a synoptophor when possible, and when not possible with a Krimsky test. All deviations were measured with appropriate spectacle correction at distance. Measurements obtained in prism dioptres were converted to degrees for data analysis. Esotropic deviations were denoted as positive numbers and exotropic deviations as negative numbers.

Refractive errors were determined with retinoscopy after cycloplegia using either cyclopentolate or atropine. The spherical equivalent values were taken into account. Myopic refraction errors were represented as negative numbers and hyperopic refraction errors as positive numbers.

Visual acuities were determined by Snellen or illiterate $\mathrm{E}$ chart when possible and by the forced choice preferential looking test when not possible. Amblyopia was defined as a difference of two or more lines between the monocular visual acuities. ${ }^{3}$ We defined the presence of 5 or more lines of difference between monocular visual acuities as deep amblyopia. Patients were divided into three groups according to the presence and degree amblyopia: group 1, patients without amblyopia; group 2, patients with amblyopia; group 3, patients with deep amblyopia.

The angle of strabismus measured at sixth postoperative week was taken for data analysis. We included only those patients whose post-operative deviations were reproducible, accurate and stable.

We included 140 esotropic and 51 exotropic patients satisfying our selection criteria.

\section{RESULTS}

Seventy-nine esotropic patients with a post-operative deviation below 5 degrees of straight at the sixth post-operative week were assigned to the success

Table I. Means and standard deviations of independent variables used in discriminant analysis for esotropic and exotropic patients

\begin{tabular}{|c|c|c|c|c|}
\hline \multirow[b]{2}{*}{ Independent variables } & \multicolumn{2}{|c|}{ Esotropic patients } & \multicolumn{2}{|c|}{ Exotropic patients } \\
\hline & $\begin{array}{l}\text { Success } \\
(n=79)\end{array}$ & $\begin{array}{l}\text { Residual esotropia } \\
(n=61)\end{array}$ & $\begin{array}{l}\text { Success } \\
(n=28)\end{array}$ & $\begin{array}{l}\text { Residual exotropia } \\
\quad(n=23)\end{array}$ \\
\hline 1. Age of onset (years) & $2.9 \pm 1.8$ & $3.8 \pm 6.8$ & $3.5 \pm 5.1$ & $2.8 \pm 2.5$ \\
\hline 2. Age at operation (years) & $7.6 \pm 3.8$ & $11.1 \pm 8.9$ & $11.8 \pm 9.3$ & $11.4 \pm 9.5$ \\
\hline 3. Interval between onset and operation (years) & $4.7 \pm 3.6$ & $7.3 \pm 6.4$ & $8.2 \pm 8.5$ & $8.7 \pm 8.9$ \\
\hline 4. Pre-operative deviation (degrees) & $14.6 \pm 7.4$ & $23.7 \pm 8.8$ & $-16.7 \pm 8.2$ & $-16.8 \pm 12.6$ \\
\hline 5. Medial rectus surgery recession/resection $(\mathrm{mm})$ & $4.6 \pm 0.5$ & $4.9 \pm 0.5$ & $5.8 \pm 1.6$ & $5.9 \pm 1.1$ \\
\hline 6. Lateral rectus surgery resection/recession $(\mathrm{mm})$ & $5.6 \pm 1.0$ & $6.4 \pm 1.0$ & $5.8 \pm 1.0$ & $6.2 \pm 0.9$ \\
\hline 7. Refractive error, right eye (dioptres) & $3.0 \pm 1.7$ & $2.4 \pm 3.1$ & $0.3 \pm 2.0$ & $0.8 \pm 1.5$ \\
\hline 8. Refractive error, left eye (dioptres) & $2.9 \pm 1.8$ & $2.5 \pm 3.1$ & $-0.8 \pm 1.9$ & $0.7 \pm 1.7$ \\
\hline 9. Anisometropia (dioptres) & $0.5 \pm 0.7$ & $0.5 \pm 0.7$ & $0.8 \pm 1.4$ & $0.3 \pm 0.3$ \\
\hline 10. Visual acuity, right eye & $0.7 \pm 0.3$ & $0.7 \pm 0.38$ & $0.8 \pm 0.3$ & $0.8 \pm 0.3$ \\
\hline 11. Visual acuity, left eye & $0.7 \pm 0.3$ & $0.6 \pm 0.3$ & $0.6 \pm 0.3$ & $0.8 \pm 0.2$ \\
\hline
\end{tabular}


Table II. Means and standard deviations of independent variables used in discriminant analysis for long-term results of esotropic patients

\begin{tabular}{|c|c|c|}
\hline Independent variables & Success $(n=49)$ & Residual esotropia $(n=32)$ \\
\hline 1. Age of onset (years) & $2.8 \pm 1.7$ & $4.5 \pm 9.1$ \\
\hline 2. Age at operation (years) & $7.5 \pm 3.7$ & $9.9 \pm 10.5$ \\
\hline 3. Interval between onset and operation (years) & $4.7 \pm 3.4$ & $5.3 \pm 5.6$ \\
\hline 4. Pre-operative deviation (degrees) & $15.5 \pm 8.5$ & $22.3 \pm 9.6$ \\
\hline 5. Medial rectus recession $(\mathrm{mm})$ & $4.7 \pm 0.5$ & $4.9 \pm 0.4$ \\
\hline 6. Lateral rectus resection $(\mathrm{mm})$ & $5.8 \pm 1.0$ & $6.2 \pm 1.1$ \\
\hline 7. Refractive error, right eye (dioptres) & $3.2 \pm 1.7$ & $2.2 \pm 3.7$ \\
\hline 8. Refractive error, left eve (dioptres) & $3.2 \pm 1.7$ & $2.3 \pm 3.7$ \\
\hline 9. Anisometropia (dioptres) & $0.6 \pm 0.6$ & $0.4 \pm 0.5$ \\
\hline 10. Visual acuity, right eye & $0.7 \pm 0.3$ & $0.7 \pm 0.3$ \\
\hline 11. Visual acuity, left eye & $0.7 \pm 0.3$ & $0.6 \pm 0.3$ \\
\hline
\end{tabular}

group. The remaining 61 patients with a deviation of more than 5 degrees of straight were assigned to the residual esotropia group. Cosmetic success for these patients was $86 \%$. For the exotropic patients 28 of 51 with a deviation below 5 degrees at the sixth postoperative week were assigned to the success group and the remaining 23 patients to the residual exotropia group. Cosmetic success for the exotropic patient group was $94 \%$. The mean and standard deviation values of independent variables used for disciminant analysis are summarised in Table I for esotropic and exotropic patients. Amblyopia was not present in $42(30 \%)$ estropic and $21(41 \%)$ exotropic patients whereas $64(46 \%)$ estropic and $21(41 \%)$ exotropic patients were amblyopic and $34(24 \%)$ esotropic and $9(18 \%)$ exotropic patients had deep amblyopia. With respect to the presence of alphabetical pattern, $52(37 \%)$ estropic and $12(23 \%)$ extropic patients had alphabetical pattern.

Among these independent variables the preoperative deviation was the only statistically significant discriminant factor for a patient being in the success group at the sixth post-operative week $(p<0.001)$. For exotropic patients the visual acuity of the left eye was statistically significant $(p<0.05)$.

In the long-term results we included 81 esotropic patients who had a post-operative follow-up longer than 1 year. Forty-nine of them with a post-operative deviation within 5 degrees of straight were assigned to the success group. The means and standard deviations of same independent variables are given in Table II. When entered into discriminant analysis the pre-operative deviation was the only statistically significant variable affecting the outcome $(p<0.05)$. We could not evaluate the long-term results for exotropic patients because there were only 19 patients who had a follow-up longer than 1 year; this patient number was not sufficient to analyse 13 independent variables with the discriminant analysis method.

In the second part of the study we analysed the factors influencing response to surgery. The mean response to surgery was $1.20 \pm 0.05$ for esotropic and $1.00 \pm 0.07$ for exotropic patients. The means and standard errors of the independent variables for esotropic and exotropic patients are given in Table III.

For esotropic patients the effect of pre-operative deviation on response to surgery was found to be statistically significant $\left(p<0.0001, r^{2}=0.33\right)$ in the first step of the multiple regression analysis. In patients with esotropia approximately $33 \%$ of the variability in response can be explained by the pre-operative deviation alone. After pre-operative deviation was put into function, age of onset of strabismus is found to be statistically significant $\left(p<0.0001, r^{2}=0.44\right)$. At the third step, when pre-operative deviation and age of onset was put into function, the amount of medial rectus recession was found to be statistically significant $\left(p<0.0005, r^{2}=0.50\right)$. At this last step these three parameters explain $50 \%$ of the variability in response. This analysis showed that response to surgery was higher for larger pre-operative deviations but lower for older age of onset of deviation and larger amounts of medial rectus recession. Figs. 1-3 show response as a function of pre-operative

Table III. The means and standard errors of variables used in multiple regression analysis for esotropic and exotropic patients

\begin{tabular}{|c|c|c|}
\hline Independent variables & Esotropic patients $(n=140)$ & Exotropic patients $(n=51)$ \\
\hline 1. Age of onset (years) & $3.0 \pm 0.3$ & $3.1 \pm 0.5$ \\
\hline 2. Age at operation (years) & $8.8 \pm 0.5$ & $11.6 \pm 1.3$ \\
\hline 3. Interval between onset and operation (years) & $5.8 \pm 0.4$ & $8.4 \pm 1.2$ \\
\hline 4. Pre-operative deviation (degrees) & $18.6 \pm 0.7$ & $-17.7 \pm 1.1$ \\
\hline 5. Medial rectus surgery recession/resection $(\mathrm{mm})$ & $4.7 \pm 0.0$ & $5.8 \pm 0.1$ \\
\hline 6. Lateral rectus surgery resection/recession $(\mathrm{mm})$ & $5.9 \pm 0.1$ & $6.0 \pm 0.1$ \\
\hline 7. Refractive error, right eye (dioptres) & $2.8 \pm 0.1$ & $0.5 \pm 0.2$ \\
\hline 8. Refractive error left eve (dioptres) & $2.7 \pm 0.1$ & $0.2 \pm 0.2$ \\
\hline 9. Anisometropia (dioptres) & $0.5 \pm 0.1$ & $0.5 \pm 0.1$ \\
\hline 10. Visual acuity, right eye & $0.6 \pm 0.2$ & $0.7 \pm 0.0$ \\
\hline 11. Visual acuity, left eye & $0.6 \pm 0.0$ & $0.7 \pm 0.01$ \\
\hline
\end{tabular}




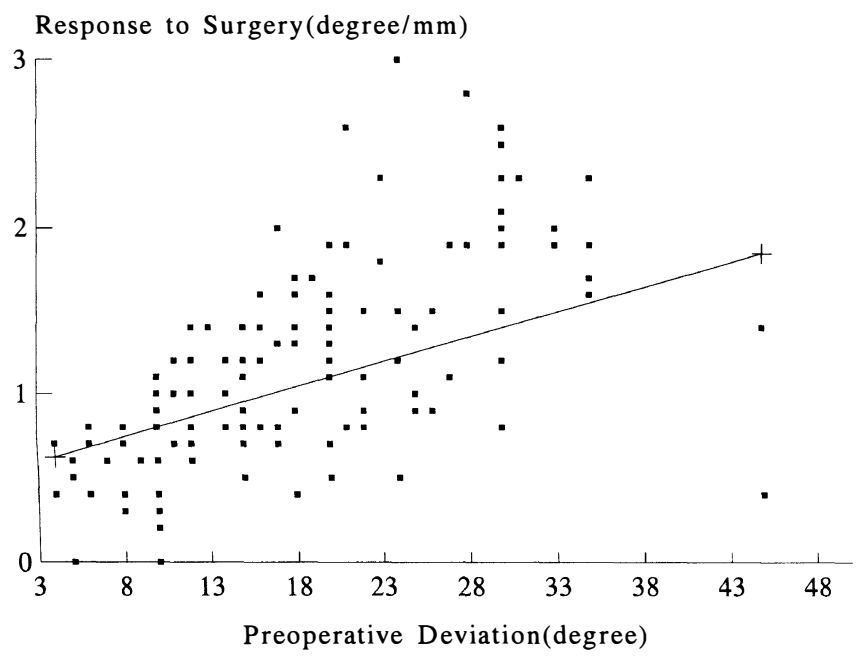

Fig. 1. Distribution of pre-operative deviations of esotropic patients as a function of response to surgery. The line represents the regression equation of the relation: Response to surgery $=0.50+(0.03 \times$ pre-operative deviation $)$.

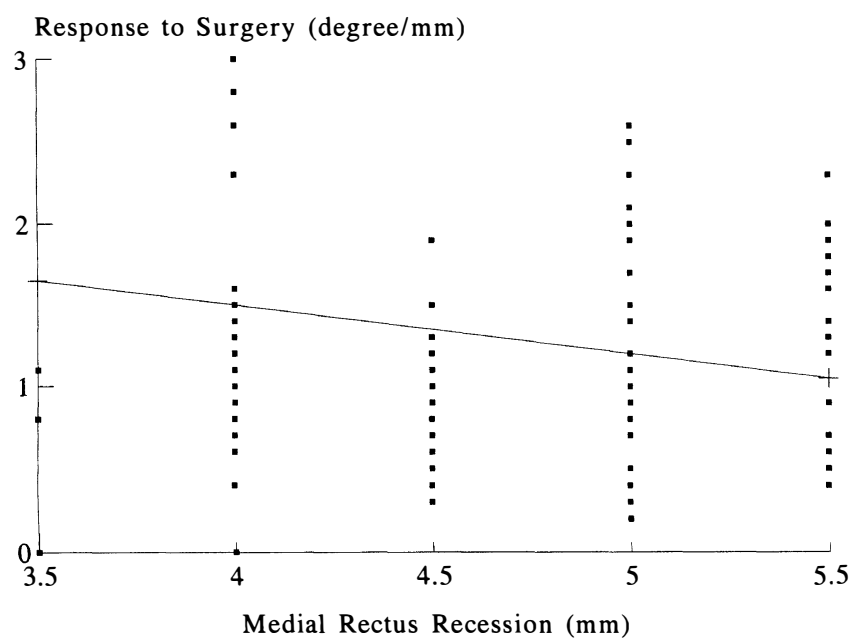

Fig. 3. Distribution of amount of medial rectus recession as a function of response to surgery for esotropic patients. The line represents the regression equation of the relation: Response to surgery $=1.78+(0.05 \times$ pre-operative deviation $)-(0.04 \times$ age of onset $)-(0.30 \times$ medial rectus recession). Pre-operative deviation is taken as 18.6 and age of onset as 3.0 (mean values).

deviation, age of onset and medial rectus recession respectively.

For 51 exotropic patients only the effect of the preoperative deviation on response to surgery was found to be statistically significant $\left(p<0.0005, r^{2}=0.25\right)$. In patients with exotropia approximately $25 \%$ of the variability in response can be explained by the preoperative deviation. The response to surgery was higher for larger pre-operative deviations in exotropic patients. Fig. 4 shows the response to surgery as a function of pre-operative deviation in exotropic patients.

\section{DISCUSSION}

In this study the pre-operative deviation was the most important discriminant factor for early and late

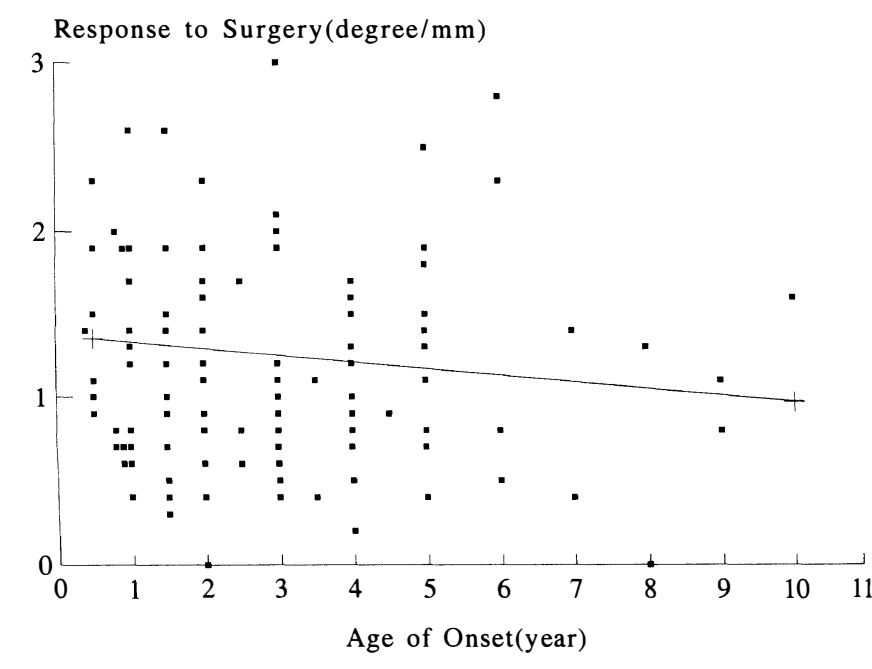

Fig. 2. Distribution of age of onset of deviation as a function of response to surgery for esotropic patients. The line represents the regression equation of the relation: Response to surgery $=0.51+(0.04 \times$ pre-operative deviation $)-(0.04 \times$ age of onset $)$. Pre-operative deviation is taken as 18.6 (mean pre-operative deviation).

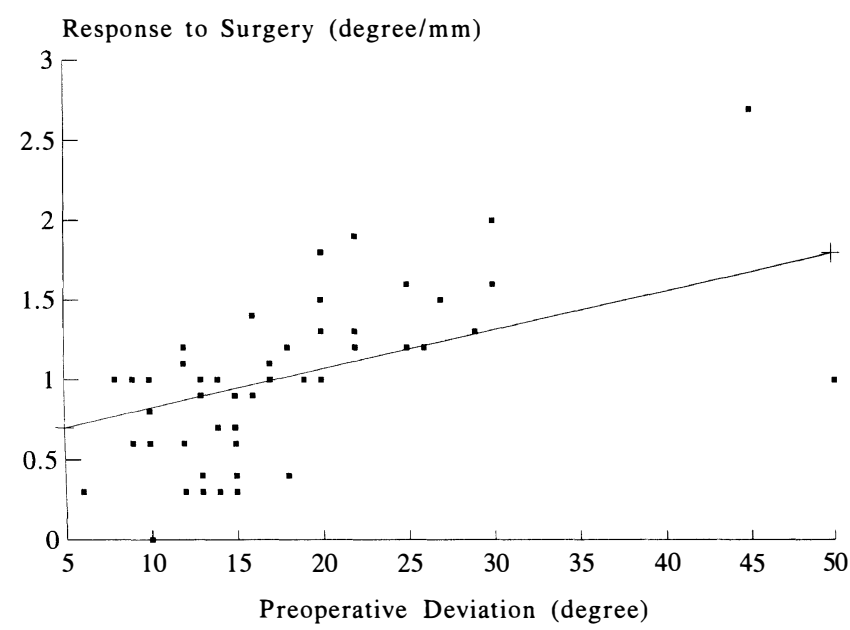

Fig. 4. Distribution of pre-operative deviations of exotropic patients as a function of response to surgery. The line represents the regression equation of relation: Response to surgery $=0.58+(0.02 \times$ pre-operative deviation $)$.

successful surgical outcomes for esotropic patients. Patients with larger pre-operative deviations have a poorer chance of having a successful outcome with a single recess-resect procedure.

Bateman and associates ${ }^{16}$ analysed variables such as age of onset of deviation, age at surgery, interval between onset of deviation and surgery, pre-operative deviation, AC/A ratio, medial rectus recession, refraction of the right and the left eyes and anisometropia in their 172 patients with discriminant analysis. They have found that the pre-operative deviation and the refractive error of the left eye were statistically significant. In another study Keenan and Willshaw $^{12}$ reported that none of the pre-operative variables, including age of onset, age at surgery, 
presence of amblyopia, refractive error and anisometropia, could explain the success of the final outcome. Their study did not include pre-operative deviation as a pre-operative variable.

For exotropic patients the only discriminant predictor for a successful outcome was the visual acuity of the left eye. Pre-operative deviation, which was the only discriminant factor for esotropic patients, was not a significant factor for exotropic patients. This may stem from the variations in measurements of pre-operative deviation depending on the examination conditions. Also the post-operative drifts are important in assigning patients to successful outcome groups. Larger patient groups with proper preoperative evaluation of exotropic patients, such as measurements with prism adaptation or monocular occlusion, may help find more significant predictors for successful surgical outcomes.

In the second part of the study we evaluated the factors influencing response to surgery. In esotropic patients the response to surgery was found to be $1.20 \pm 0.05$ degree $/ \mathrm{mm}$ (range $0-3$ degree $/ \mathrm{mm}$ ). Multiple regression analysis had shown that the response to surgery increased with increasing amounts of pre-operative deviation. But it was lower for patients with older age of onset and larger amounts of medial rectus recession. The relationship between pre-operative deviation and response has also been demonstrated previously. ${ }^{17-19}$ It was suggested by Graf and associates ${ }^{17}$ that this was related to the presence of anomalous correspondence in small angles of deviation. They suggested that anomalous correspondence is of no consequence in very large deviations, which results in large postoperative angles, but plays an increasing role in small pre-operative deviations. Also, in larger recessions of the medial rectus muscle the recessed muscle postoperatively inserts more dorsal to the tangential point than pre-operatively.

Kushner and associates ${ }^{18}$ reviewed factors influencing response to surgery and stated that the inclusion of axial length, age or refractive error did not significantly improve the prediction of response beyond that provided by the use of pre-operative deviation alone.

Our study showed that the older the patient at the onset of deviation the smaller the surgical effect. This can be explained by the fact that the younger the patient, the smaller the eye and the larger the response. This relation was also demonstrated by Kuschner and associates. ${ }^{18}$

In this study the response to surgery was found to be lower for larger amounts of medial rectus recessions. This was an unexpected result because larger amounts of medial rectus recession were done for larger pre-operative deviations and response was greater in larger pre-operative deviations. This result may stem from the anatomical variations in the insertions of the recti muscles. The limbus-insertion and insertion-equator distance are important for function of the recessed medial rectus muscle postoperatively. It has been shown that limbus-equator distance for the medial rectus can vary betwen 9.10 and $13.76 \mathrm{~mm}$, while the amount of recession needed to place the medial rectus at the equator ranged between 3.5 and $8.5 \mathrm{~mm}^{20}$ Another minor contributing factor can be the use of compasses for intraoperative measurements of recessions instead of curved rulers. ${ }^{21}$ The effect may be more pronounced in larger recessions.

For exotropic patients the response was found to be $1.00 \pm 0.07$ degrees $/ \mathrm{mm}$ surgery. The only factor influencing response to surgery was the pre-operative deviation. The response was higher for larger preoperative deviations. There is no consensus on the factors influencing response in exotropic patients. Scott $e t$ al. $^{22}$ found age, the difference between near and distance deviations and the change in deviation with upward and downward gaze to be significant predictors influencing response in exotropic patients. Gordon and Bachar, ${ }^{6}$ using the same predictor variables, found that response was determined by the magnitude of the larger pre-operative deviation, the average corrected visual acuity, the degree of anisometropia and the average spherical equivalent. Kuschner and associates ${ }^{18}$ demonstrated that the pre-operative deviation significantly influenced response in exotropic patients while Graf and associates $^{18}$ asserted that it did not. The lack of agreement in these results may be due to the greater variation of pre-operative angles of deviation in exotropic patients. The use of the pre-operative prism adaptation test for determining the preoperative deviation in exotropic patients was shown to improve the surgical outcome. ${ }^{23}$ Also it is recommended that the deviations be measured after 1 hour of monocular occlusion in exotropic patients. ${ }^{24}$ Another factor creating discrepancy between the results of different series may be the post-operative drift encountered in exotropic patients. ${ }^{25}$

These factors are not sufficient to explain the variability in response to strabismus surgery. Several other pre-, intra- and post-operative factors are suggested to play a role in the final outcome of surgery. The most important of these may be the errors made in measuring the pre-operative deviations. This may be due to the examiner or the prismatic effect of spectacles having a refractive power greater than \pm 5 dioptres $^{26}$ and incorrect conversion of prism dioptres to degrees. ${ }^{7.27}$ The use of prism adaptation for determining the pre-operative angle of deviation is recommended both for esotropic and exotropic patients. ${ }^{14,23}$ 
One of the intraoperative errors in recession surgery is the placement of sutures about $1 \mathrm{~mm}$ beyond the insertion line, which may decrease the effective amount of recession. ${ }^{7}$ The posterior dislocation of the central part of the recessed or resected muscle may increase the effect of recession while decreasing the effect of resection. Recently Rosenbaum and associates ${ }^{28}$ reported that the size of the pre-operative deviation and response to surgery were correlated with the passive tension of the medial rectus muscle in patients with acquired esotropia. ${ }^{28}$

Post-operatively fibrosis, atrophy, paresis and cicatrisation may affect the outcome. ${ }^{7.29}$ The astigmatism and other refractive changes after strabismus surgery also contribute to the final outcome. ${ }^{30}$

All these factors are important in the final results of strabismus surgery. Pre-operative deviation was found to be the strongest predictor for response to strabismus surgery for both esotropic and exotropic patients. It was also the only discriminant factor for a successful surgical outcome in esotropic patients. We believe that eliminating the factors causing errors in the determination of pre-operative deviation will improve the success and predictability of surgical outcomes.

We are grateful to A. T. Moore, MA, FRCS, FRCOphth, for revising the manuscript.

Key words: Amblyopia, Esotropia, Exotropia. Strabismus surgery, Surgical response.

\section{REFERENCES}

1. von Graefe A. Beitraege zur Lehre vom Schielen und von der Schiel-Operationen. Graefes Arch Ophthalmol 1857;3:177-386. In: Simonsz HJ, Dijk BV. Analysis of the dosage controversy in recess-resect and faden surgery with the Robinson Computer Model of eye movements. Doc Ophthalmol 1988;67:237-52.

2. von Pflugk A. Beitrag zur Technik der Schieloperationen: Vornaehung und Zuruecknaehung. Ber DOG 1906;34-44. In: Simonsz HF, Dijk BV. Analysis of the dosage controversy in recess-resect and faden surgery with the Robinson Computer Model of eye movements. Doc Ophthalmol 1988;67:237-52.

3. von Noorden GK. Binocular vision and ocular motility: theory and management of strabismus. St Louis: CV Mosby, 1990.

4. Scobee RG. Esotropia: incidence, etiology, and results of therapy. Am J Ophthalmol 1951;34:817-33.

5. Gillies WE, Hughes A. Results in 50 cases of strabismus after graduated surgery designed by A scan ultrasonography. Br J Ophthalmol 1984;68:790-5.

6. Gordon YJ, Bachar E. Multiple regression analysis predictor models in exotropia surgery. Am J Ophthalmol 1980;90:687-91.

7. Simonsz HJ, Dijk BV. Analysis of the dosage controversy in recess-resect and faden surgery with the Robinson Computer Model of eye movements. Doc Ophthalmol 1988;67:237-52.

8. Vazquez RL. The effects of surgical technique and the radius of the eye on correction for horizontal strabismus. Ann Ophthalmol 1987;19:187-93.
9. Sener EC, Oruc S, Abbasoglu OE, Dayanir V, Sanac AS. The effect of vertical transposition in horizontal rectus surgery. Turkish Ophthalmic Society 27th National Congress Bulletin, 1993;1:181-4.

10. Kutschke PJ, Keech RV. The effect of vertical displacement of horizontal muscles on the deviation in primary position. Am Orthop J 1988;38:117-22.

11. Kushner BJ, Fisher MR, Lucchese NJ, Morton GV. Factors influencing response to strabismus surgery. Arch Ophthalmol 1993;111:75-9.

12. Keenan JM, Willshaw HE. The outcome of strabismus in childhood esotropia. Eye 1990;7:341-5.

13. Wheeler MC. Long term results of surgical treatment of strabismus. J Pediatr Ophthalmol 1972;9:76-8.

14. Prism Adaptation Study Research Group. Efficacy of prism adaptation in the surgical management of acquired esotropia. Arch Ophthalmol 1990;108: 1248-56

15. Edwards WC, Moran CT. Askew W. Statistical analysis of esotropia surgery. J Pediatr Ophthalmol 1973; 10:256-66.

16. Bateman B, Parks M, Wheeler N. Discriminant analysis of acquired esotropia surgery. Ophthalmology 1983;90:1154-9.

17. Graf M, Krzizok T, Kaufmann H. The influence of axial length on the effect of horizontal strabismus surgery. Binocular Vision 1993;8:233-40.

18. Kushner BJ, Lucchese NJ, Morton GV. The influence of axial length on the response to strabismus surgery. Arch Ophthalmol 1989;107:1616-8.

19. Romano PE. Essay. Eye muscle surgery dosimetry: an international comparison and pseudo-paradox regarding power. Binocular Vision 1993;8:281-2.

20. Kushner BJ, Lucchese NJ, Morton GV. Variation in axial length and anatomical landmarks in strabismic patients. Ophthalmology 1991;98:400-6.

21. Scott WE, Martin-Casals A, Braverman DE. Curved ruler for measurement along surface of globe. Arch Ophthalmol 1978;96:1084-6.

22. Scott AB, Mash AJ, Jampolsky A. Quantitative guidelines for exotropia surgery. Invest Ophthalmol 1975;14:428-31.

23. Ron A, Merin S. The use of the pre-op prism adaptation test (PAT) in the surgery of exotropia. Am Orthop J 1988;38:107-10.

24. Cooper J, Medow N. Major review: intermittent exotropia basic and divergence excess type. Binocular Vision 1993:8:185-216.

25. Maruo T, Kuboto N, Iwashige H, Kamiya Y. Longterm results after strabismus surgery. Graefes Arch Clin Exp Ophthalmol 1988;226:414-7.

26. Scattergood KD, Brown MH, Guyton DL. Artifacts introduced by spectacle lenses in the measurement of strabismic patients. Am J Ophthalmol 1983;96:439-48.

27. Thompson JT, Guyton DL. Ophthalmic prisms: measurement errors and how to minimise them. Ophthalmology 1983;90:204-10.

28. Rosenbaum AL, Egbert JE, Keogan T, Wheeler N, Wang C, Buzard K. Length-tension properties of extraocular muscles in patients with esotropia and intermittent exotropia. Am J Ophthalmol 1994; 117:791-9.

29. Robinson DA. A quantitative analysis of extraocular muscle cooperation and squint. Invest Ophthalmol 1975;2:801-20.

30. Thompson WE, Reinecke RD. The changes in refractive status following routine strabismus surgery. J Pediatr Ophthalmol Strabismus 1984;17:372-4. 